\section{THE ATLAS TRIGGER AND DATA ACQUISITION SYSTEM}

The ATLAS Trigger and DAQ System reduces the LHC collision rate to an affordable recording rate It is composed by a Level1 (L1) hardware implemented on custom build electronics and an High Level Trigger (HLT) implemented in software on a large PC farm. HLT runs on fine grained data and is divided in:

OLevel2 (L2) where fast and ad-hoc designed algorithms run on a limited portion of the event read

Event Filter (EF) where algorithms imported from the offline reconstruction run on full reconstructed events.

The performance of the ATLAS TDAQ system is exceeding its design Event Rates

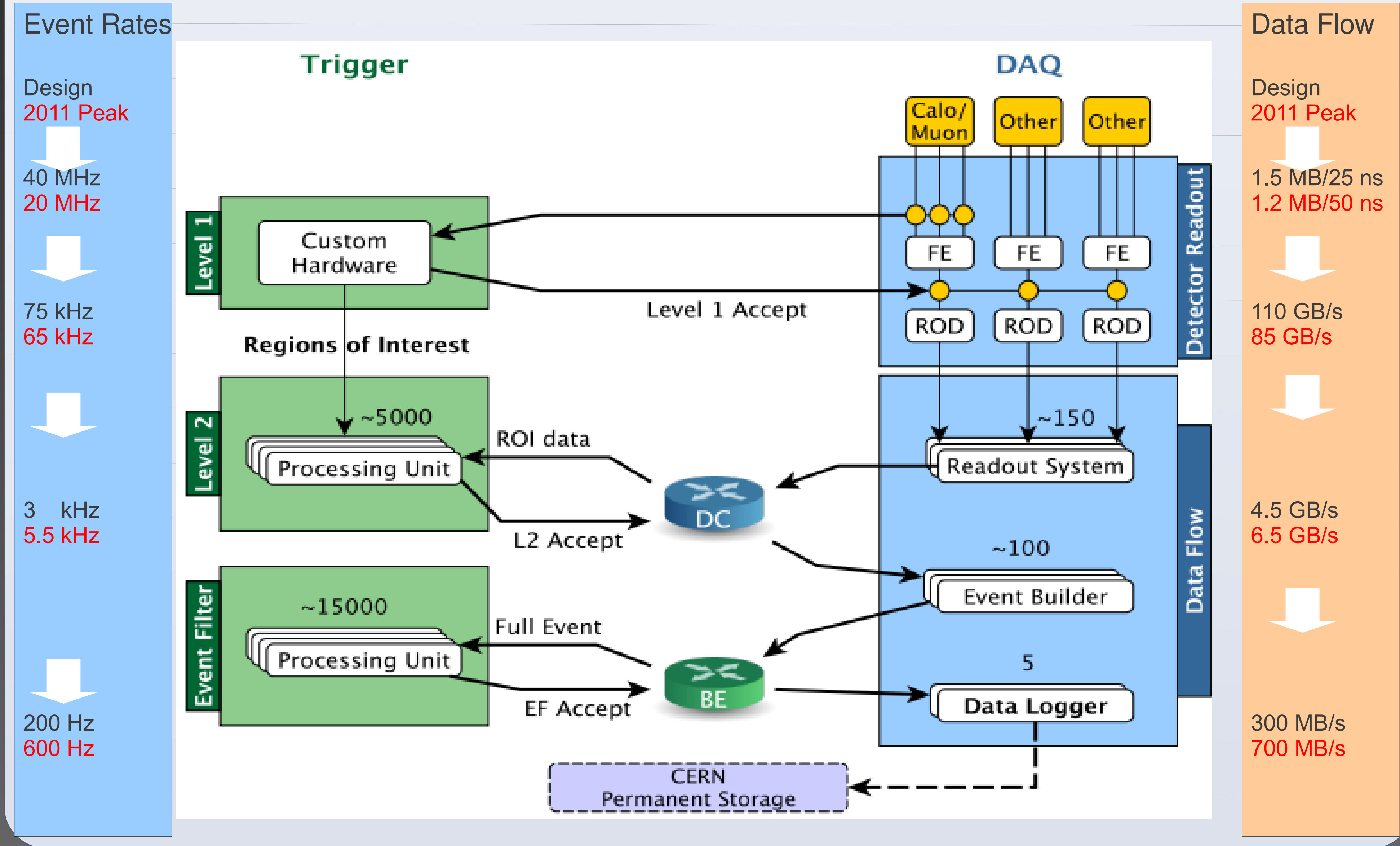

LHC AND ATLAS: 2011 VS 2012

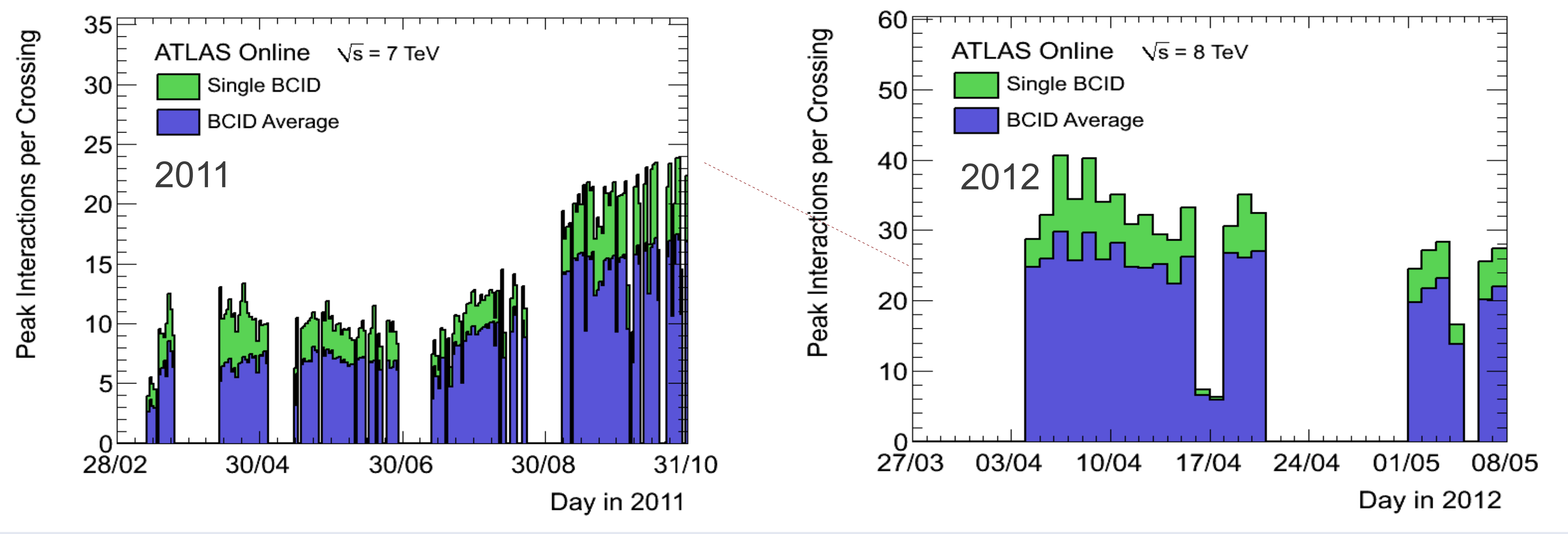

Pile up $<\mu>$ has constantly increased during 2011 and 2012 causing:

Rapid rate increase for low momentum objects (i.e. more then linearly)

Running with $\sqrt{s}=8 \mathrm{TeV}$ in 2012 increases the rates by $\sim 20 \%$ because of the production cross section Runcese

TDAQ rate capability was gained with a limited improvement of the hardware. Read Out Server (ROS) refurbishing allowing increase the access rate from $\mathrm{HLT}$ farms from $22 \mathrm{kHz}$ to $\sim 40 \mathrm{kHz}$

OReplacement of $16 \mathrm{HLT}$ racks with more performing processing nodes and more cores

\section{Trigger selections are organized in ingER SIGNATURES}

physics signatures: e. Electron/gamma, muon, physics signatures: e.g. Electron/gamma, muon,
tau, jet, MissingET, B-jet, B-physics, MinBias

Lowest threshold unprescaled trigger per signatures are usually the primary trigger selection for physics measurements

In 2011 although the luminosity was increasing, With 2012 further luminosity increase TDAQ had to modify trigger selections

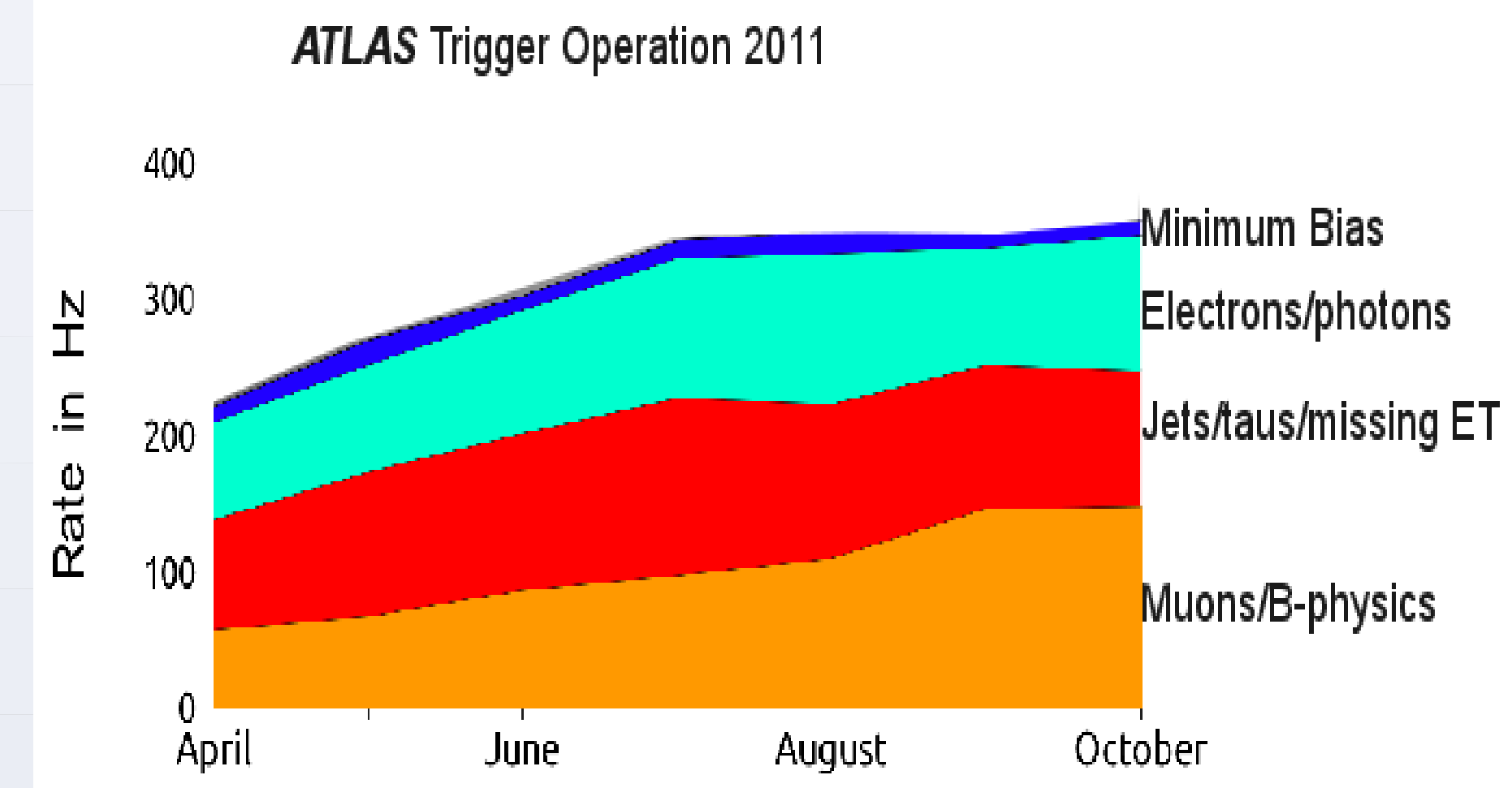

-Small increase of momentum thresholds. Larger increase are not possible since they would reduce too much the efficiency.

(electron/gamma) and isolation (electron/muon). Since ere are up sensitive, their effect has to be carefully checked.

\begin{tabular}{|c|c|c|c|c|}
\hline \multicolumn{5}{|c|}{ Thresholds of the lowest unprescaled chain selection per signature. } \\
\hline \multirow[b]{2}{*}{$\begin{array}{l}\text { Trigger Signatures } \\
\text { (multiplicities) }\end{array}$} & \multicolumn{2}{|c|}{2011} & \multicolumn{2}{|c|}{2012} \\
\hline & $\begin{array}{l}\text { L1 Thresholds } \\
(\mathrm{GeV})\end{array}$ & $\begin{array}{l}\text { HLT Thresholds } \\
(\mathrm{GeV})\end{array}$ & $\begin{array}{l}\text { L1 Thresholds } \\
(\mathrm{GeV})\end{array}$ & $\begin{array}{l}\text { HLT Thresholds } \\
(\mathrm{GeV})\end{array}$ \\
\hline Electron (1) & 16 , had & $20 / 22$ had & $\begin{array}{l}18, \text { had } \\
30\end{array}$ & $\begin{array}{l}24 \text {, had, iso } \\
60\end{array}$ \\
\hline Electron (2) & $2 \times 10$, had & $2 \times 12$ & $2 \times 10$, had & $2 \times 12$, had \\
\hline Gamma (1) & 30 & 80 & 30 & 120 \\
\hline Gamma (2) & $2 \times 12$ & $2 \times 20$ & $\begin{array}{l}2 \times 10, \text { had } \\
12+16\end{array}$ & $\begin{array}{l}2 \times 20, \text { had } \\
30+20\end{array}$ \\
\hline \multirow{2}{*}{ Muon (1) } & \multirow{2}{*}{11} & \multirow{2}{*}{18} & 15 & 24 iso \\
\hline & & & 15 & 36 \\
\hline Muon (2) & $2 \times 4$ & $2 \times 10$ & $2 \times 6$ & $2 \times 13$ \\
\hline Tau (1) & 30 & $100 / 125$ & 40 & 125 \\
\hline Tau (2) & $2 \times 11$ & $29+20$ & $11+15$, iso & $29+20$, iso \\
\hline Jet (1) & 75 & 240 & 75 & 360 \\
\hline Jet (5) & $5 \times 10$ & $5 \times 30$ & $4 \times 15$ & $45 \times 55$ \\
\hline MET (Global) & 50 & 60 & $60(50)$ & 80 \\
\hline
\end{tabular}

\section{SUMMARY}

The ATLAS TDAQ system worked with excellent performances in 2011. To keep the same achievements in 2012, with more severe LHC conditions (pile up and luminosity), some improvements had to be
implemented. First, tighter algorithms have been used, having in mind that they need to be robust against pile up. Then, a continuous campaign of smooth hardware upgrades allowed to run TDAQ with larger rates.
LEVEL 1

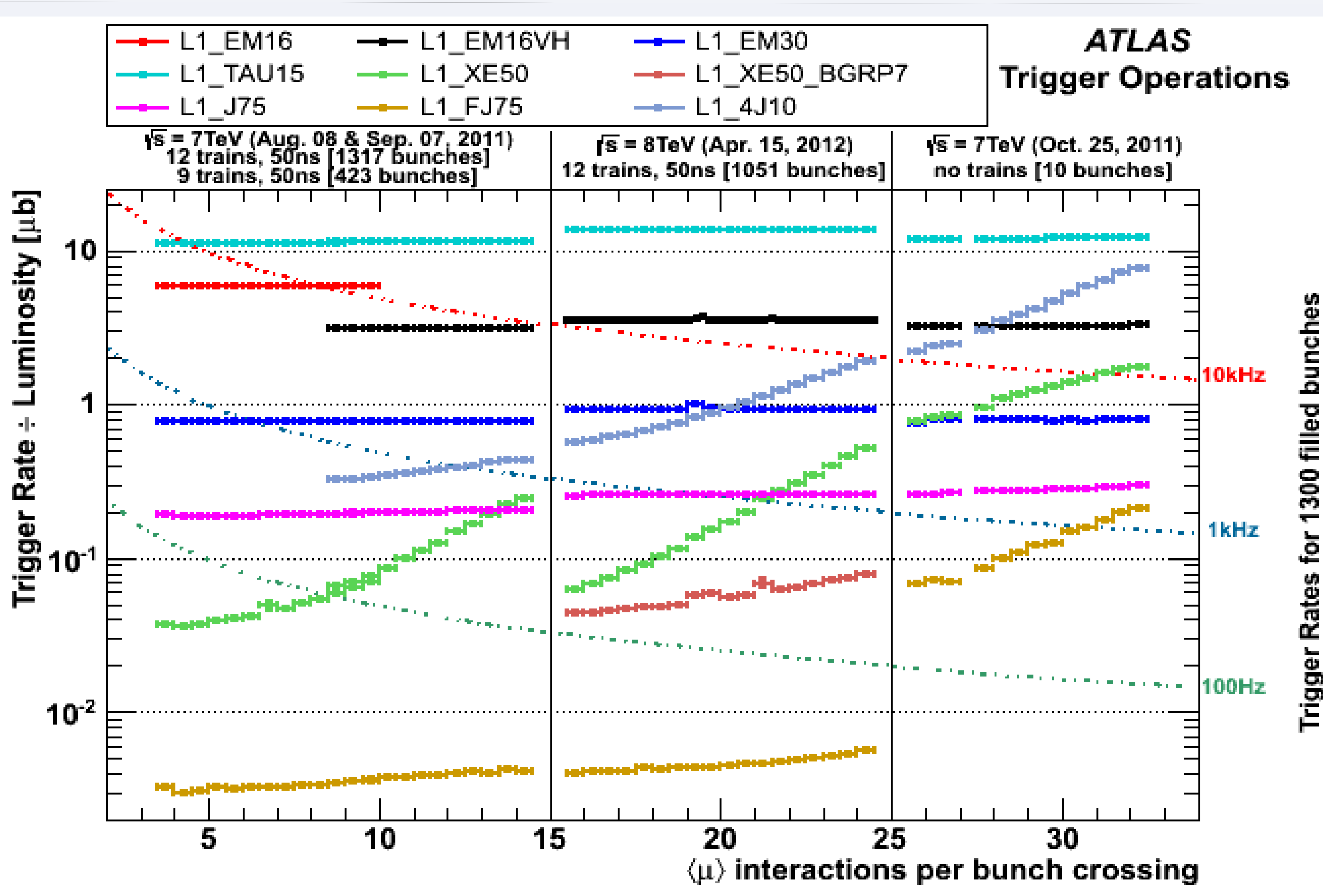

L1 Trigger cross sections vs pile up (2011 and 2012 data). The chains affected by pile up are mostly Many efforts in 2012 to keep L1 rates under control

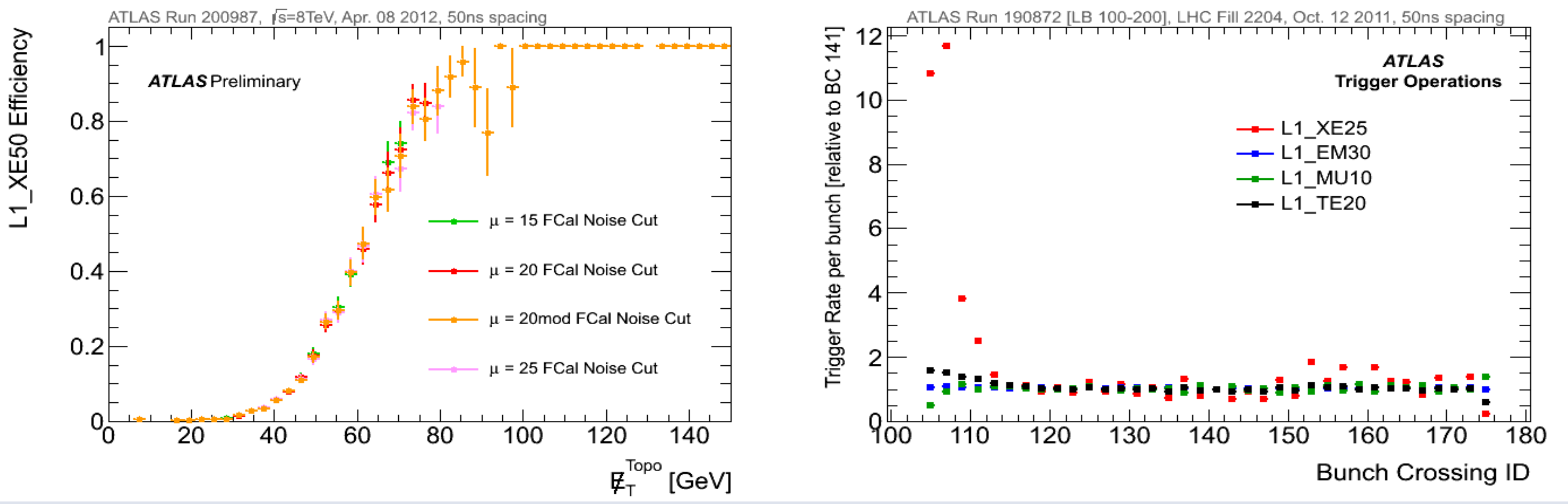

Main improvements for 2012 .

Applied stronger calorimeter noise cuts in particular in the forward regions $\rightarrow$ Checks that L1 efficiencies are only marginally $<\mu>$ dependent

Some $\mathrm{L} 1$ triggers ( $\mathrm{L} 1$ XE25 which requires $\mathrm{ME} \mathrm{T}_{\mathrm{T}}>25 \mathrm{GeV}$ ) have significant larger rates near the start of bunch trains due to the unbalanced overlaying of bipolar calorimeter trigger signals shapes from neighboring bunches $\rightarrow$ Veto triggers on first bunches with a small impact on luminosity collected ( $\sim 8 \%)$ from Front-End electronics Boards allowing a larger $L 1 M E_{T}$ rate input to $H L T$.

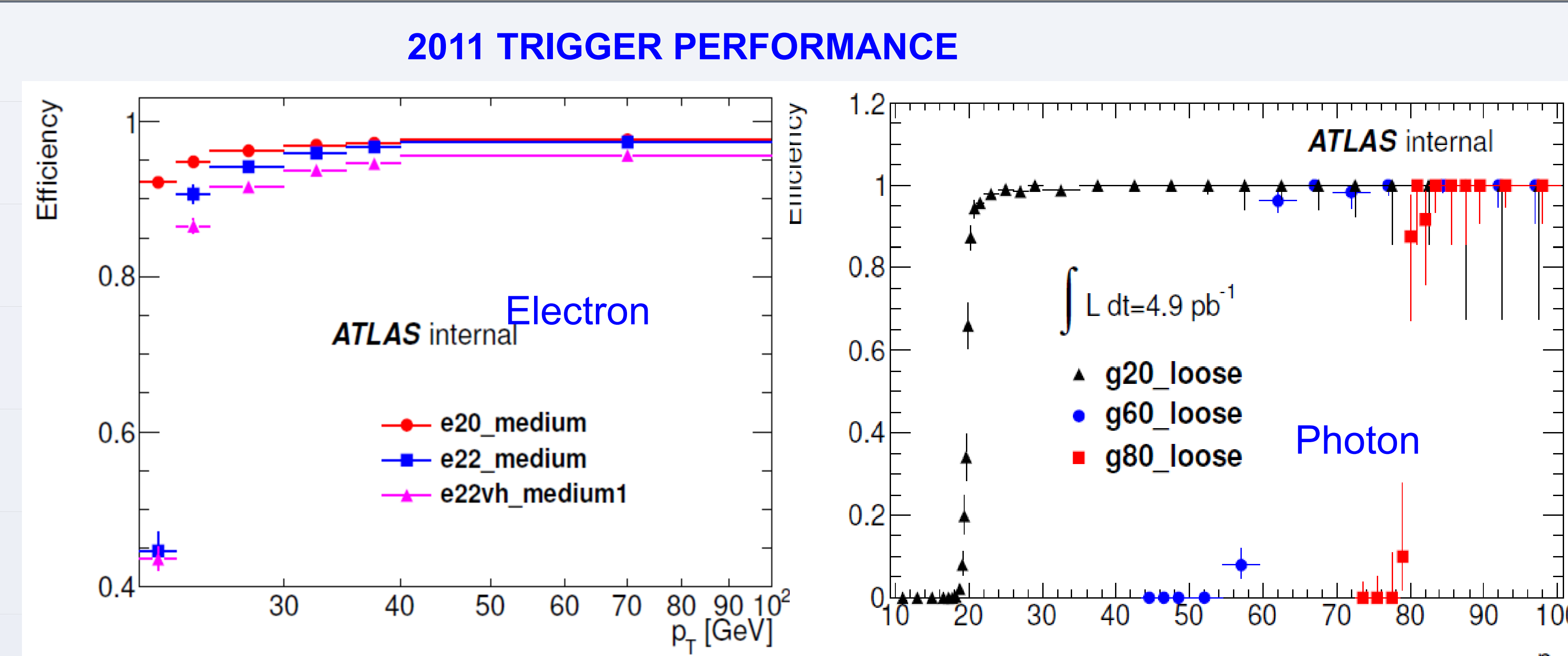

Trigger efficiencies are measured from data wrt offline selection. They are displayed as a function of offline reconstructed $\mathrm{P}_{\mathrm{T}} / \mathrm{E}_{\mathrm{T}}$

Electron and Muon trigger efficiencies are measured with the Tag \& Probe method (with $\mathrm{Z} \rightarrow \mathrm{ee}, \mathrm{Z} \rightarrow \boldsymbol{\mu} \mu$ ) OPhoton efficiencies are measured with the bootstrap method . First, the L1 efficiency of events collected by an orthogonal trigger (muon,jet,minimum bias) is measured wrt offline selection cuts. Then the HLT
selection efficiency is measured wrt events that pass

$\mathrm{L} 1$ and offline requirements such that:
Eff (gammaloffline) $=$

Eff(L1|offline\&MinBias) x Eff(HLT|L1\&offline)

Muon trigger plateau efficiency is limited by geometrical acceptance

Muon and electron trigger efficiencies are marginally affected by pile up than ID tracking isolation)

Electron and muon trigger efficiencies are marginally affected by pile up (further improved in 2012)
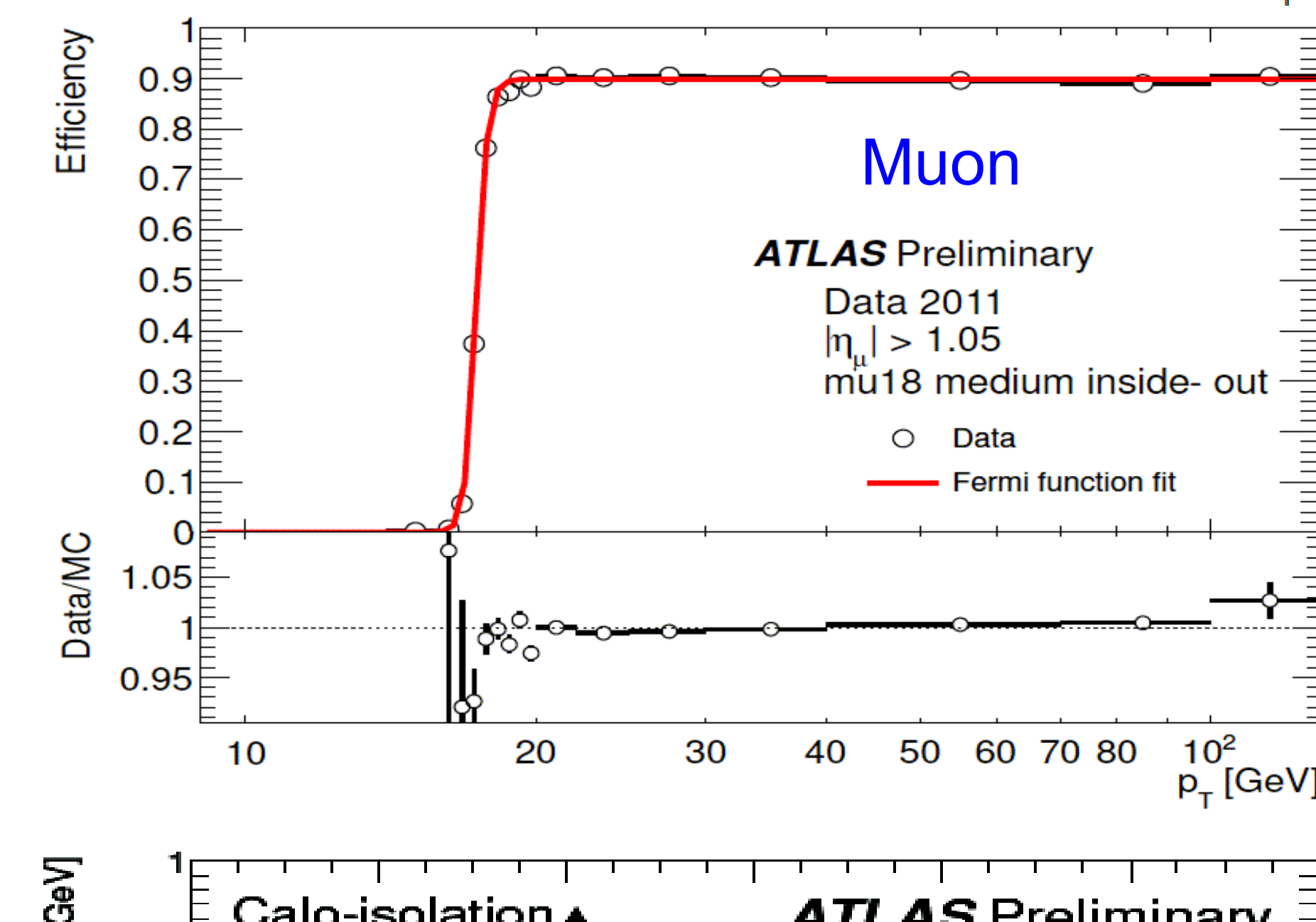

0.9 Calo-isolation 1 ATLAS Preliminary •
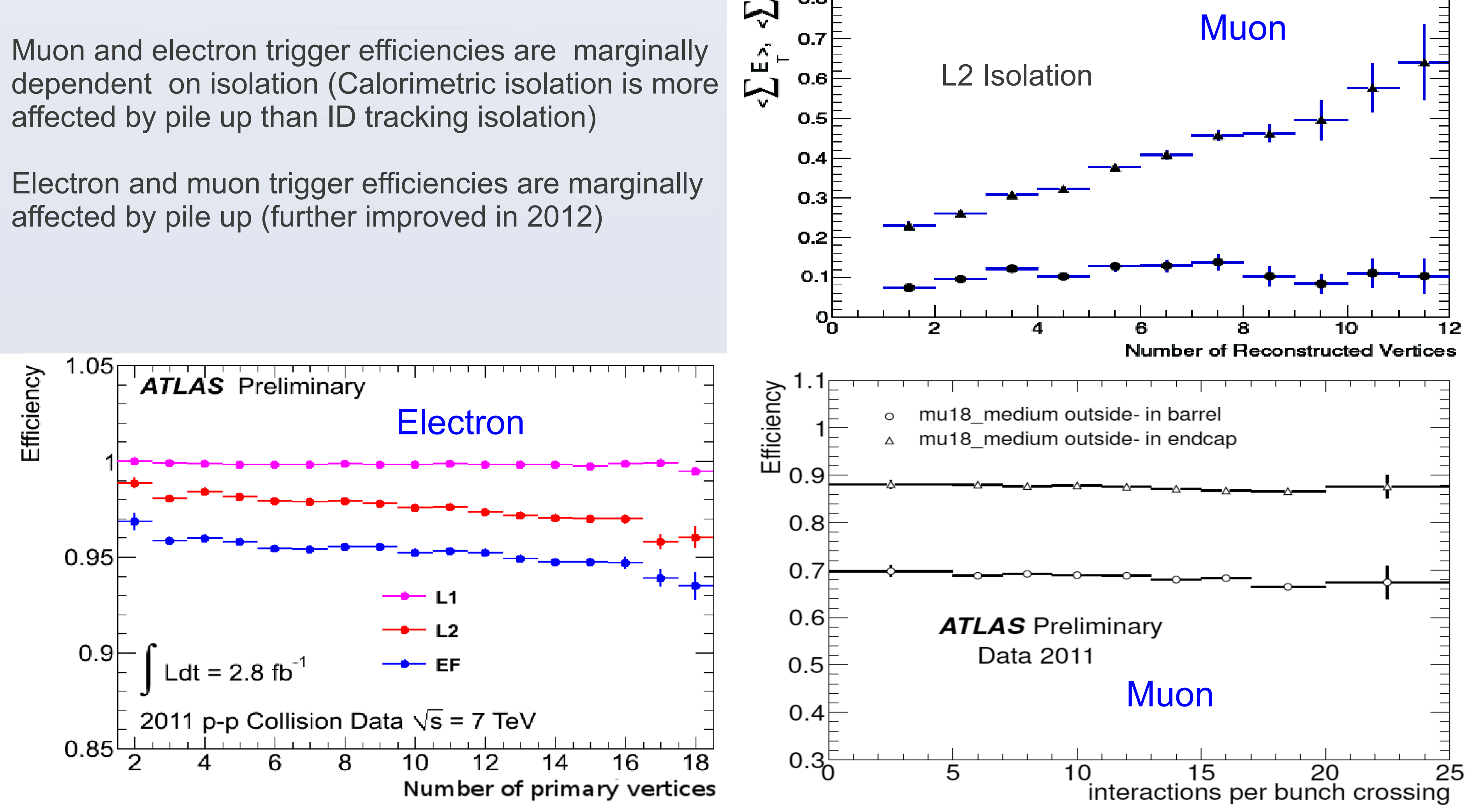